REVIEW

\title{
The Early Repolarization ECG Pattern - An Update
}

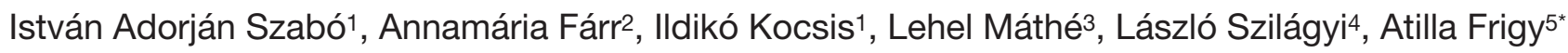 \\ 1. Department of Cardiology, Clinical County Hospital Mures, Tîrgu Mureș, Romania \\ 2. Department of Physiopathology, University of Medicine and Pharmacy of Tîrgu Mureș, Romania \\ 3. Department of Internal Medicine VII, University of Medicine and Pharmacy of Tîrgu Mureș, Romania \\ 4. Sapientia University, Tîrgu Mureș, Romania \\ 5. Department of Internal Medicine IV, University of Medicine and Pharmacy of Tîrgu Mureș, Romania
}

Early repolarization pattern (ERP), a form of J-wave syndromes, was considered long time a benign ECG phenomenon. However, recent data confirmed that certain phenotypes of ERP are related to an increased risk of sudden cardiac death (idiopathic ventricular fibrillation). Our paper gives a short and practical update regarding the main issues related to ERP: epidemiological data, molecular and electrophysiological background, clinical significance and risk stratification. At the end, the future directions of research and clinical management related to ERP are presented.

Keywords: J-wave, early repolarization syndrome, sudden cardiac death

Received 3 August 2017 / Accepted 10 October 2017

\section{Introduction. History}

From the beginning of electrocardiography, so called idiopathic J-waves were found on the ECG recordings of many species, including humans. The J-point on ECG is defined as the transition between the end of the QRS complex and the ST segment. Deviation of this point from the isoelectric line determine the presence of J-deflection (wave), which could be followed by upsloping or horizontal ST-segment elevation, determining the appearance of early repolarization ECG pattern (ERP). The ERP was first described in 1936 by Shipley and Hallaran, who studied 200 young healthy men and women and described the Jdeflection as notching of the descending part of the QRS complex [1-3].

ERP was considered previously as a benign, "innocent", ECG phenomenon, as a distinct form of the so called J-wave syndromes (which include also the Brugada syndrome). However, in the last ten years, epidemiological and case-control studies found some forms of ERP to be predictors and risk factors for sudden cardiac death (primary ventricular fibrillation). This is particularly true, when the ST segment has a horizontal or descending direction in the inferior and/or infero-lateral leads. The risk is even higher when the amplitude of J-wave is greater than $0.2 \mathrm{mV}$ [2].

\section{Diagnostic criteria. Prevalence}

Several consensus papers were issued on diagnostic criteria of ERP. The last criteria are summarized in Table I [4].

Early repolarization syndrome is a term used for those patients who present ERP on the 12-lead ECG and had a previous episode of aborted sudden cardiac death or had

\footnotetext{
* Correspondence to: Atilla Frigy
}

E-mail: afrigy@rdslink.ro documented malignant ventricular arrhythmias (ventricular fibrillation, polymorphic ventricular tachycardia) [2].

The prevalence of ERP in the general population is between $1 \%$ and $9 \%$, but is $15 \%$ to $70 \%$ in patients with idiopathic ventricular fibrillation. Observing different patient cohorts, ERP produced an increase of 4 to 10 fold in the occurrence of sudden cardiac death, the maximal incidence being reached between 35 and 45 years $[5,6]$.

Regarding the influence of gender, ERP is strongly associated with male sex, men representing more than $75 \%$ of cases. Also, in male subjects a greater J-point elevation was observed than in women, and males represent $75 \%$ of the malignant cases. Surawicz et al. set a hormonal hypothesis after evaluating the ECGs of 529 males aged 5 to 96 years. They observed that the prevalence of ERP increased parallelly with the rise of testosterone levels during puberty. In elderly males, when testosterone levels are in decline, the prevalence of ERP is decreased $[5,7]$.

ERP is more common in young and physically active individuals, a phenomenon which regress with age. In black people this pattern is particularly prevalent, however, these subjects were underrepresented in diverse studies and their arrhythmic risk is still undetermined. In athletes ERP shows a higher prevalence than in the general population: a presence in $20 \%$ of noncompetitive and almost $90 \%$ in competitive athletes $[5,6]$.

\section{Table I. Current diagnostic criteria of ERP [4]}

1 Presence of J-wave - notching at the end of the QRS complex (Fig. 1) or slur on the positive R-wave descending limb (Fig. 2), with or without ST-segment elevation

$2 \mathrm{~J}$-wave peak amplitude greater than $0.1 \mathrm{mV}$, present in $\geq 2$ contiguous leads, except the $\mathrm{V} 1-\mathrm{V} 3$

3 QRS duration less than $120 \mathrm{~ms}$ in the leads not containing notching or slur 

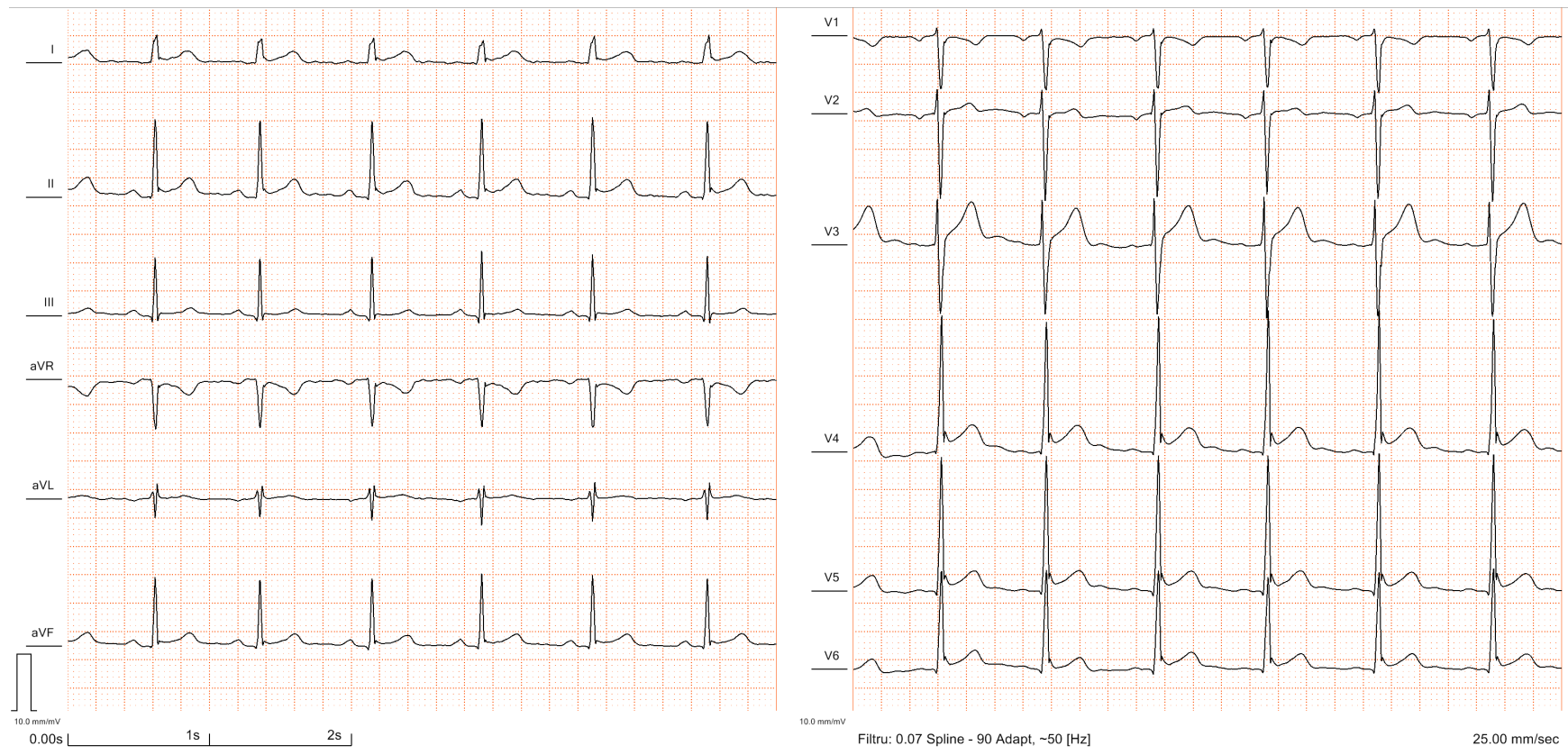

Fig. 1. ECG registration with J-waves appearing as notching at the end of the QRS complex, best visible in V4-6 leads (from the personal collection of Dr. Frigy A.)
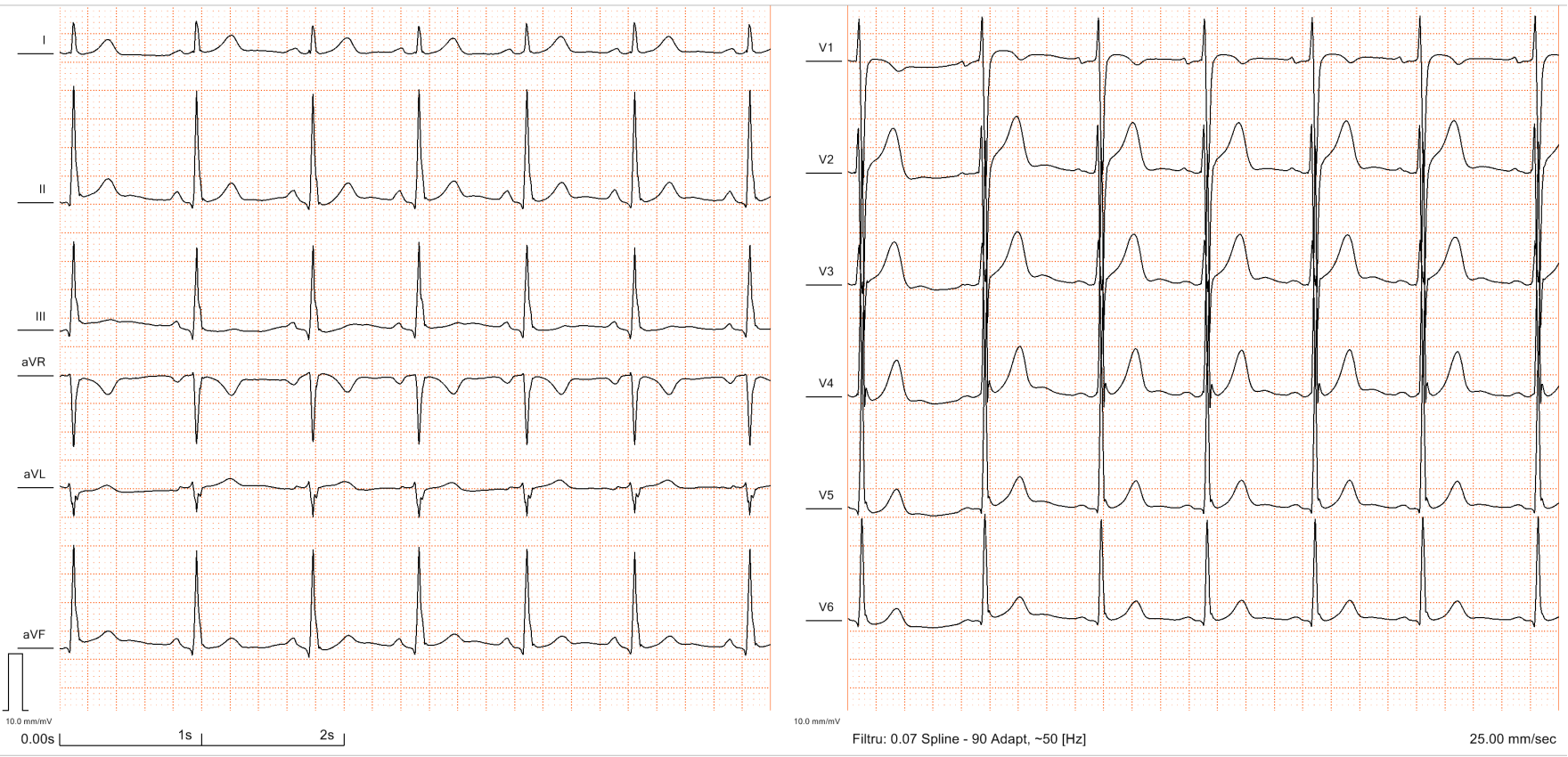

Fig. 2. ECG registration with J-waves appearing as slur on the positive R-wave descending limb in infero-lateral (II, III, aVF, V5,6) leads (from the personal collection of Dr. Szabó I.A.)

\section{Molecular basis. Electrophysiological background}

Antzelevitch et al. in the 1980s performed an experiment in canine, founding a heterogeneous transmural distribution of the transient outward potassium current $\left(\mathrm{I}_{\text {to }}\right)$ in the ventricular myocardium. Their hypothesis was the following: for the appearance and shape of the J-wave the transient outward potassium current is responsible, which, being not uniformly active in the myocardium, produces differences between the epicardial and endocardial action potential (AP) shapes [3, 8].
The normal epicardial AP of the ventricular myocardium differs from that of endocardial, having a prominent phase 1 notch or a typical spike-and-dome morphology. This is due to the fact that during the 1 st phase of AP there is a large $I_{\text {to }}$ in the epicardium, producing a more intense initial repolarization. In the case of early repolarization there is an increase in the difference in AP amplitude at the endocardial-epicardial level, which manifests as Jwave (Fig. 3). This wave corresponds to the current flowing from the endocardium to epicardium during phase 1. The characteristic notch on the epicardial action potential coincides with the J-wave on the surface ECG, also, they 


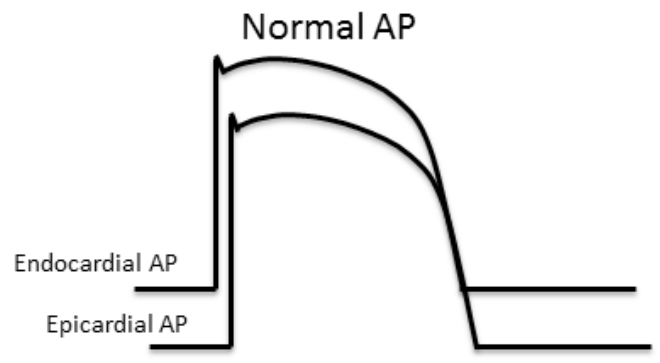

Normal ECG

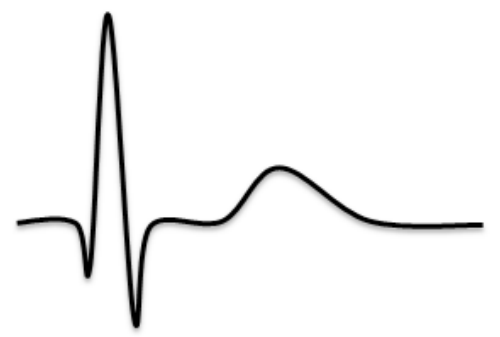

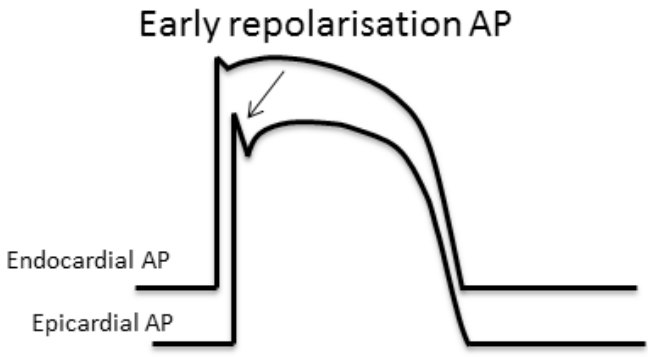

Early repolarisation ECG

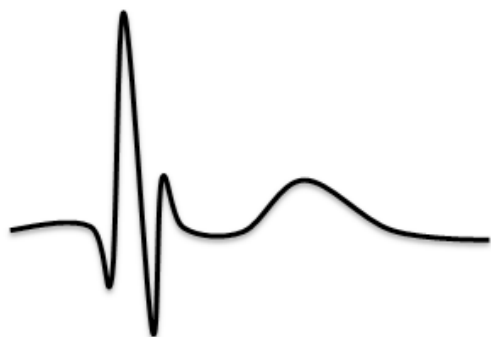

Fig. 3. Electrophysiological basis of the appearance of J-wave on surface ECG (see explanations in text) [9]

change parallelly, ventricular activation clearly influencing the appearance of J-wave $[8,9]$.

The phenomenon could be more enhanced in the setting of mutations of genes encoding inward $\mathrm{Na}^{+}, \mathrm{Ca}^{2+}$ or outward $\mathrm{K}^{+}$currents. The functional properties of these mutations have not been determined definitively. Independently of the initiating mechanism, the ventricular tachyarrhythmia is maintained by local transmural reentry induced by the increased AP gradients. The genetic basis for early repolarization syndrome is not entirely clarified, but today's data show seven different genes encoding cardiac ion channels that are associated with early repolarization syndrome, more exactly, $3 \mathrm{~K}^{+}$channel related, $3 \mathrm{Ca}^{2+}$ channel related and $1 \mathrm{Na}^{+}$channel related genes were found to be involved $[2,9]$.

The first reported gene was KCNJ8 which encodes the inward rectifying $\mathrm{K}^{+}$channel Kir6.1, one of the subunits of the cardiac ATP-sensitive $\mathrm{K}+$ channel. A gain-of-function mutation of KCNJ8 - S422L - was first identified in a 14-year-old Caucasian female who suffered of recurrent ventricular fibrillation [10].

\section{Clinical significance. Risk stratification}

ERP, known as notch or slur of the downsloping limb of the QRS complex, was considered long time a benign and innocent ECG phenomenon. The benign nature of ERP was challenged in 2000, based on experimental data showing that the presence of this ECG manifestation predisposes to the development of polymorphic ventricular tachycardia and ventricular fibrillation [11]. Validation of this hypothesis was provided 8 years later by Haissaguerre et al., Nam et al., and Rosso et al. These studies, together with other case-control and population-based studies demonstrated the increased risk for development of malignant arrhythmias and sudden cardiac death in patients having ERP, especially localized in the inferior and infero-lateral leads. However, the appearance of J-waves on a routine, screening ECG does not have automatically the significance of "high risk" for malignant arrhythmic events, because the odds for ventricular fibrillation are as low as $1: 10,000$. Rosso et al. stated that the occurrence of a J wave on the surface ECG increases the probability of ventricular fibrillation from 3.4:100,000 to $11: 100,000$. [11-15] It is important to mention, that there are emerging data concerning the ventricular arrhythmia promoting effect of ERP in patients with chronic coronary heart disease and in the setting acute myocardial infarction $[16,17]$.

The most difficult task in managing ERP is risk stratification, the correct and feasible estimation of the risk of sudden cardiac death, to distinguish those features which

Table II Genetic background of ERP and early repolarization syndrome - the main genes involved and the corresponding ion channels [2]

\begin{tabular}{|c|c|c|c|c|}
\hline & Gene & Protein & Ion channel & $\%$ of probands \\
\hline & KCNJ8 & Kir6.1 & IK-ATP & 0.6 \\
\hline \multirow[t]{3}{*}{ K+ channel } & ABCC9 & SUR2A & IK-ATP & 7.3 \\
\hline & KCND2 & $\mathrm{Kv} 4.2$ & Ito & 2.0 \\
\hline & CACNA1C & Cav1.2 & Ica & 4.1 \\
\hline \multirow[t]{2}{*}{ Ca 2+ channel } & CACNB2b & $\operatorname{Cav} \beta 2 b$ & Ica & 8.3 \\
\hline & CACNA2D1 & Cava2d & Ica & 4.1 \\
\hline $\mathrm{Na}+$ channel & SCN5A & Nav1.5 & $\mathrm{INa}$ & 6.0 \\
\hline
\end{tabular}


Table III. Schematic representation of the elements of risk stratification in patients with ERP. [2] Abbreviations: VF - ventricular fibrillation, VT - ventricular tachycardia, SCD - sudden cardiac death, VPBs - ventricular premature beats

\begin{tabular}{|c|c|c|}
\hline & \multicolumn{2}{|c|}{ J-WAVE MANIFESTATIONS } \\
\hline & Topography (ECG leads) & Clinical and other ECG features \\
\hline 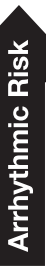 & $\begin{array}{l}\text { - Global presence } \\
\text { - Right precordial (Brugada ECG pattern) } \\
\text { - Inferior or infero-lateral } \\
\text { - Lateral }\end{array}$ & $\begin{array}{l}\text { - Resuscitation from cardiac arrest, documented VF, or polymorphic VT } \\
\text { - Positive family history of SCD, arrhythmic syncope, gene mutations } \\
\text { - Short-coupled VPBs } \\
\text { - Fragmented QRS } \\
\text { - Short QT } \\
\text { - Dynamic changes in J-wave amplitude } \\
\text { - J-waves }>2 \mathrm{mV} \\
\text { - J-waves with horizontal /downsloping ST-segment } \\
\text { - Tall R-waves, rapidly ascending ST segment }\end{array}$ \\
\hline
\end{tabular}

are related with an increased arrhythmic risk. Table III summarizes the most important elements - and their relative value - in risk stratification [2]. Tikkanen et al., in their papers stated that a rapidly ascending ST- segment after the J point in healthy athletes, seems to be a benign variant of ERP, while a horizontal or descending ST-segment elevation is associated with an increased risk of arrhythmic death $[18,19]$.

Present guidelines recommend (class I indication) an implanted cardioverter defibrillator (ICD) in survivors of an episode of ventricular fibrillation in the case of ERP ( = early repolarization syndrome). But, we still miss firm recommendations for the treatment of patients with ERP who have only syncope of unknown origin, or are asymptomatic. The majority of patients with ERP does not require any intervention, but have to be carefully investigated. [2]

Primary prevention of sudden cardiac death in asymptomatic ERP is a major challenge. Present data suggest that a J-point elevation $>0.2 \mathrm{mV}$ linking short QTc interval confers a 3 -fold increase in sudden cardiac death risk. ICD implantation is recommended in patients with early repolarization syndrome who have survived a cardiac arrest, while quinidine may be helpful in those patients with ICD and frequent non-sustained ventricular tachyarrhythmias. In acute setting, another therapeutic approach is to increase heart rate beyond 90 , up to 120 beats/min with isoproterenol infusion, which can suppress ventricular arrhythmias. In patients who are not responsive to pharmacological treatment endocardial ablation of ectopic foci in the early repolarization zone can be useful $[2,3]$.

\section{Future perspectives. Conclusions}

The main challenge for the future in the case of ERP consists of risk stratification. This is/has to be a complex task, based on multiple parameters: clinical, ECG and genetic features, biomarkers, etc. There are some promising new methods, especially for the identification of genetic (causative mutations) and electrical substrates of the malignant forms of ERP. ECGI (electrocardiographic imaging) is a translational research tool used in the setting of inherited arrhythmia syndromes. This technique records the body surface potentials using several electrodes and computes truly the electrical activity of epicardium. [8, 20, 21]

ERP is an ECG phenomenon which still remains con- troversal in many aspects: precise diagnostic and screening criteria, complex electrophysiological and genetic background, feasible risk stratification and prophylactic treatment. For the everyday clinician is very important to observe and identify the phenomenon and to make an initial risk stratification based on history and common ECG features.

\section{Funding}

The article is part of a research project supported by the grant nr. 141/26.01.2017 of the Hungarian Academy of Science and Studium-Prospero Foundation.

\section{Conflict of interest}

None to declare.

\section{References}

1. Gussak I, Gussak G. History of the $J$ wave and $J$ wave syndromes. In: Antzelevitch C, Yan GX: J Wave Syndromes. Brugada and Early Repolarization Syndromes. Springer International Publishing Switzerland. 2016, p.1-12.

2. Antzelevitch C, Yan GX, Ackermann MJ, et al. J-wave syndromes expert consensus conference report: Emerging concepts and gaps in knowledge. Heart Rhythm. 2016; 13: e295-e324.

3. Antzelevitch C, Yan GX. J wave syndromes. Heart Rhythm. 2010; 7: 549-558.

4. Macfarlane PW, Antzelevitch C, Haissaguerre M, et al. The early repolarization pattern: a consensus paper. J Am Coll Cardiol. 2015; 66: 470-477.

5. Maury P, Rollin A. Prevalence of early repolarization/J wave patterns in the normal population. J Electrocardiol. 2013; 46: 411-416.

6. Noseworthy PA, Tikkanen JT, Porthan K, et al. The early repolarization pattern in the general population. J Am Coll Cardiol. 2011; 57: 22842289.

7. Surawicz B, Parikh SR. Prevalence of male and female patterns of early ventricular repolarization in the normal ECG of males and females from childhood to old age. J Am Coll Cardiol. 2002; 40: 1870-1876.

8. Mercer BN, Begg GA, Page SP, et al. Early repolarization syndrome; Mechanistic theories and clinical correlates. Frontiers in physiology. 2016; 7:1-12.

9. Obeyesekere MN, Klein GJ, Nattel S., et al. A clinical approach to early repolarization. Circulation. 2013; 127: 1620-29.

10. Haïssaguerre M, Chatel S, Sacher F, et al. Ventricular fibrillation with prominent early repolarization associated with a rare variant of $\mathrm{KCNJ8/}$ KATP channel. J Cardiovasc Electrophysiol. 2009; 20: 93-98.

11. Rosso R, Kogan E, Viskin S, et al. J-point elevation in survivors of primary ventricular fibrillation and matched control subjects: incidence and clinical significance. J Am Coll Cardiol. 2008; 52: 1231-1238.

12. Haïssaguerre $M$, Sacher F, Nogami $A$, et al. Characteristics of recurrent ventricular fibrillation associated with inferolateral early repolarization: role of drug therapy. J Am Coll Cardiol. 2009; 7: 612-619.

13. Nam GB, Kim YH, Antzelevitch C. Augmentation of $\mathrm{J}$ waves and electrical storms in patients with early repolarization. $\mathrm{N}$ Engl J Med. 2008; 358: 2078-2079. 
14. Rosso R, Adler A, Viskin S., et al. Risk of sudden death among young individuals with $\mathrm{J}$ waves and early repolarization: putting the evidence into perspective. HeartRhythm. 2011; 8: 923-929.

15. Adler A, Viskin S, Rosso R, et al. What Do We Know About the "Malignant Form" of Early Repolarization? J Am Coll Cardiol. 2013; 62: 863-868.

16. Patel RB, Reddy V, Chokshi M, et al. Early repolarization associated with ventricular arrhythmias in patients with chronic coronary artery disease. Circ Arrhythm Electrophys. 2010; 3: 489-495.

17. Zhang Z, Letsas KP, Yang Y, et al. Notching early repolarization pattern in inferior leads increases risk of ventricular tachyarrhythmias in patients with acute myocardial infarction: a meta-analysis. Sci Rep. 2015; 5: 15845.
18. Tikkanen JT, Junttila MJ, Anttonen O, et al. Early repolarization: electrocardiographic phenotypes associated with favorable long-term outcome. Circulation. 2011; 123: 2666-2673.

19. Junttila MJ, Sager SJ, Tikkanen JT, et al. Clinical significance of variants of J-points and J-waves: early repolarization patterns and risk. Eur Heart J. 2012; 33: 2639-2644.

20. Ghosh S, Cooper DH, Haissaguerre M, et al. Early repolarization associated with sudden death: insights from noninvasive electrocardiographic imaging. Heart Rhythm. 2010; 7: 534-537.

21. Rudy Y, Lindsay BD. Electrocardiographic imaging of heart rhythm disorders: from bench to bedside. Card Electrophysiol Clin. 2015; 7: 17-35. 